Dieses Dokument ist eine Zweitveröffentlichung (Verlagsversion) / This is a self-archiving document (published version):

Richard Mühle, Hannes Ernst, Stephan B. Sobottka, Ute Morgenstern

Workflow and hardware for intraoperative hyperspectral data acquisition in neurosurgery

Erstveröffentlichung in / First published in:

Biomedical Engineering / Biomedizinische Technik. 2020, 66 (1), S. 31-42 [Zugriff am: 29.03.2021]. De Gruyter. ISSN 1862-278X.

DOI: https://doi.org/10.1515/bmt-2019-0333

Diese Version ist verfügbar / This version is available on:

https://nbn-resolving.org/urn:nbn:de:bsz:14-qucosa2-742618 
Richard Mühle*, Hannes Ernst, Stephan B. Sobottka and Ute Morgenstern

\section{Workflow and hardware for intraoperative hyperspectral data acquisition in neurosurgery}

https://doi.org/10.1515/bmt-2019-0333

Received December 20, 2019; accepted May 27, 2020

\begin{abstract}
To prevent further brain tumour growth, malignant tissue should be removed as completely as possible in neurosurgical operations. Therefore, differentiation between tumour and brain tissue as well as detecting functional areas is very important. Hyperspectral imaging (HSI) can be used to get spatial information about brain tissue types and characteristics in a quasi-continuous reflection spectrum. In this paper, workflow and some aspects of an adapted hardware system for intraoperative hyperspectral data acquisition in neurosurgery are discussed. By comparing an intraoperative with a laboratory setup, the influences of the surgical microscope are made visible through the differences in illumination and a pixel- and wavelength-specific signal-to-noise ratio (SNR) calculation. Due to the significant differences in shape and wavelength-dependent intensity of light sources, it can be shown which kind of illumination is most suitable for the setups. Spectra between 550 and 1,000 nm are characterized of at least $40 \mathrm{~dB}$ SNR in laboratory and $25 \mathrm{~dB}$ in intraoperative setup in an area of the image relevant for evaluation. A first validation of the intraoperative hyperspectral imaging hardware setup shows that all system parts and intraoperatively recorded data can be evaluated. Exemplarily, a classification map was generated that allows visualization of measured properties of raw data. The results reveal that it is possible and beneficial to use HSI for
\end{abstract}

\footnotetext{
*Corresponding author: Richard Mühle, Faculty of Electrical and Computer Engineering, Institute of Biomedical Engineering, Technische Universität Dresden, 01062 Dresden, Germany; Department of Neurosurgery, Faculty of Medicine Carl Gustav Carus, Technische Universität Dresden, 01307 Dresden, Germany, E-mail: richard.muehle@tu-dresden.de. https://orcid.org/00000002-6831-8942

Hannes Ernst and Ute Morgenstern: Faculty of Electrical and Computer Engineering, Institute of Biomedical Engineering, Technische Universität Dresden, 01062 Dresden, Germany, E-mail: hannes.ernst@tu-dresden.de (H. Ernst), ute.morgenstern@tudresden.de (U. Morgenstern)

Stephan B. Sobottka: Department of Neurosurgery, Faculty of Medicine Carl Gustav Carus, Technische Universität Dresden, 01307 Dresden, Germany, E-mail: stephan.sobottka@uniklinikumdresden.de
}

wavelength-related intraoperative data acquisition in neurosurgery. There are still technical facts to optimize for raw data detection prior to adapting image processing algorithms to specify tissue quality and function.

Keywords: brain tumour surgery; hyperspectral imaging; intraoperative image data acquisition; neurosurgery; optical imaging; optical tissue parameters.

\section{Introduction}

Despite the latest technology in neurosurgery, individually adapted brain tumour resection is one of the biggest challenges in this field. It is necessary to eliminate tumour tissue, to prevent further malignant tissue growth, but to preserve functional intact brain tissue. The overall survival for patients after surgery is directly connected with the extent of brain tumour resection [1, 2]. Because of ambition to preserve healthy tissue in order to avoid restriction of brain functions, differentiation between tumour and brain tissue as well as detecting functional areas is an important technological task in neurosurgery. Based on computed tomography (CT), magnetic resonance imaging (MRI), functional magnetic resonance imaging (fMRI), and positron emission tomography (PET), tissue quality can be specialized preoperatively [3]. State-of-the-art for intraoperative orientation of surgeons during brain tumour resection is multifaceted. Surgeons use methods like 5-ALA fluorescence imaging, intraoperative MRI with diffusion tensor imaging (DTI), 3D ultrasound imaging, electrophysiological functional mapping after stimulation, intraoperative optical imaging (IOI) after peripheral or direct electrical cortical stimulation (DCS). Preoperative data, microscopy, and further data acquired during brain tumour resection are fused intraoperatively via neuronavigation system. Thus, surgeons provide essential assistance, but navigation deteriorates in accuracy, when altered pressure conditions after craniotomy cause brain shift [4]. Imaging after peripheral stimulation allows to identify active functional brain areas. Detecting IOI stimulation answers after direct electrical cortical stimulation allows to differentiate between tumour and non-tumour tissue [4-8].

Medical prior knowledge about different tumour types, their optical characteristic and practical 
experiences from IOI enable combining hyperspectral imaging (HSI) with IOI during neurosurgical interventions. For example, fast growing tumours have a high lactate concentration which points anaerobic glycolysis, followed by increased $\mathrm{O}_{2}$ consumption [9]. On the one hand, higher oxygenation indicates malignant tissue at pathologic angiogenesis and hypermetabolism. For another kind of brain tumour, an indication of malignancy is the significantly lower oxygenation of tissue under examination, compared to healthy tissue [10]. Both types result in altered $\mathrm{O}_{2}$ consumption and abnormal haemoglobin concentration [11].

The scheme of a three-dimensional data cube, acquired by an HSI camera (TIVITA Tissue Camera, Diaspective Vision $\mathrm{GmbH}$, Germany) and the neurosurgical HSI setup used are shown in Figure 1. The camera registers tissue reflectance with a resolution of $\lambda=5 \mathrm{~nm}$ concerning to each pixel of the surface area projection image. These characteristics of all substances contained in the upper tissue layers yield more information about the chemical tissue composition and differences to other tissues than the optical image from surgical microscope.

HSI cameras are working in different wavelength ranges: from ultraviolet $\left(\lambda_{\mathrm{UV}}=200-380 \mathrm{~nm}\right)$ to infrared $\left(\lambda_{\mathrm{IR}}=2,500-10,000 \mathrm{~nm}\right)$ range [11]. Some HSI systems use two cameras with different wavelength ranges [12], and others use only one camera for the lower wavelength range $\left(\lambda_{\text {VIS-NIR }}=500-1,000 \mathrm{~nm}\right)$ [13]. In general, there are three main methods for acquisition of hyperspectral imaging data. Point or line scanning methods use a whisk broom or push broom technology. In contrast, spectral scanning captures the entire scene simultaneously for one wavelength band. Further wavelength ranges are recorded one by one until the entire wavelength range has been captured $[14,15]$.

Most groups working with intraoperative HSI systems deal with abdominal cavity [16]. Jansen-Winkeln et al. [17], dealing with surgical questions in the intestine, have successfully used HSI to determine resection margin of colorectal resections instead of macroscopic evaluation. During the HELICoiD project, a demonstrator is developed with two HSI cameras, separate lighting unit, scanning platform and positioning camera. They showed that it is possible to detect human brain tumours in neurosurgery $[12,18]$.

However, most of today's experimental HSI systems have, among other things, due to their size and complexity of their construction, major problems in hardware and implementation in everyday clinical practice which need to be optimized. They require separate attachments for lighting, stepper motor and camera, cannot be covered sterilely, generate additional work for the clinical staff or

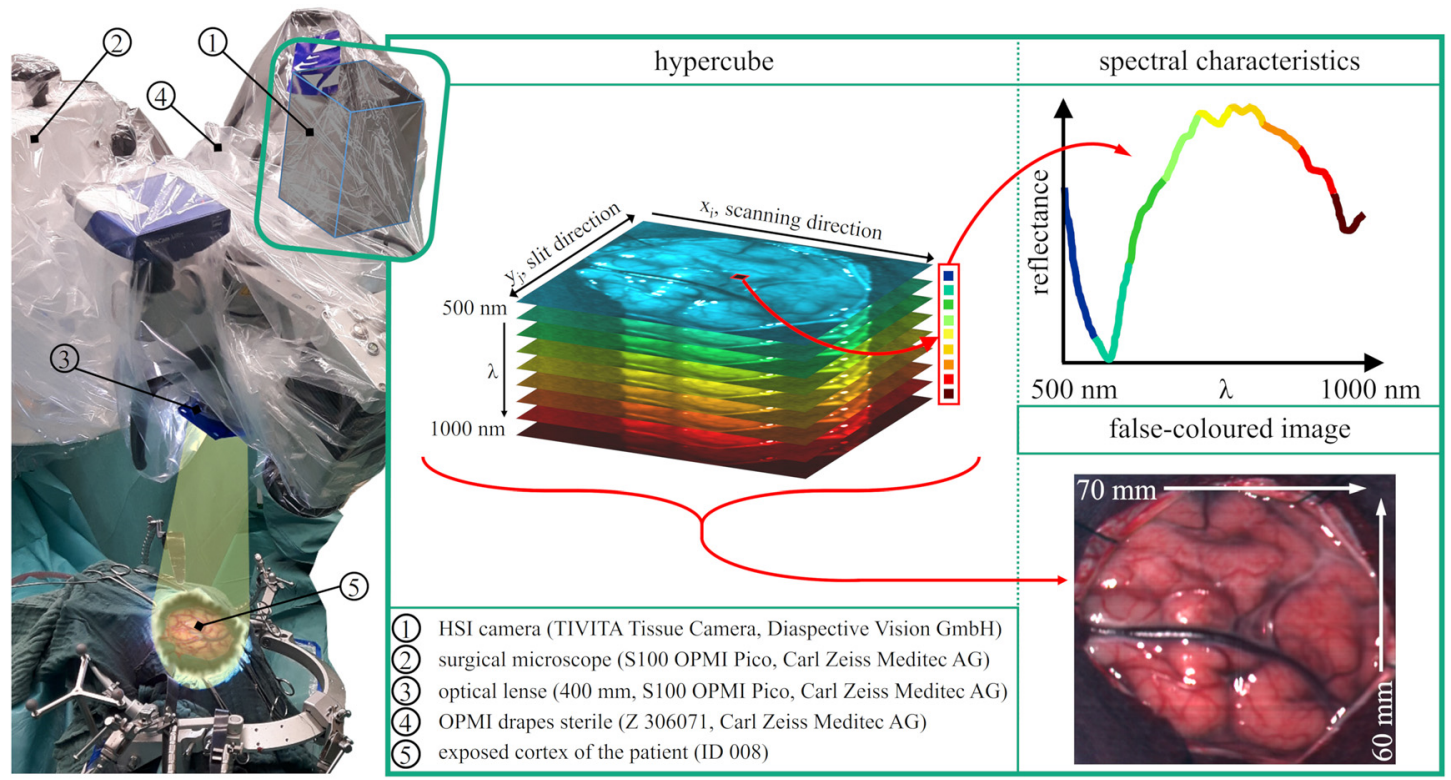

Figure 1: Structure of a hyperspectral imaging (HSI) three dimensional data cube. The neurosurgical HSI setup (left) consists of a HSI camera (1), a surgical microscope (2), the optical lenses (3), a sterile drape (4) and the exposed cortex of a patient (5). The data cube, relating to the $x-y-$ image (bottom right, patient ID008) of the trepanned area from surgical microscope (left) consists of a series of two dimensional images for each resolved wavelength, shown in the middle. The reflectance curve of one selected pixel $\left(x_{i}, y_{j}\right)$ from one of these $x$-y-images characterizing the cube is displayed on the top right. With this neurosurgical HSI setup the maximum recorded trepanned area can reach $60 \times 70 \mathrm{~mm}$ (bottom right). 
cannot be integrated quickly and easily into the clinical routine. The systems cannot be integrated into the intraoperative microscope. For this reason, every HSI measurement has to be planned carefully into the course of a tumour resection and cannot be performed spontaneously during an ongoing operation [12, 17-19].

To develop an HSI system that can be well integrated into the clinical workflow, the following technical requirements has to be met: The HSI camera has to be small and not rely on external stepper motors to move the slit, on additional illumination or setups. In addition, the HSI-camera has to be coupled to the same beam path of the microscope like illumination and the ocular for surgeon. The most important step is to integrate an optimized powerful light source on the microscope with a spectral characteristic matched to the HSI camera.

Each component that is implemented into the beam path of an HSI recording setup has influence on quality of the measurement. That is why we need to know the optical characteristics of all setup subcomponents in order to assess their influence on the outcome of the system.

In this work, our knowledge about integration of optical imaging in neurosurgery is used to integrate HSI into the workflow of a neurosurgical brain tumour resection. The aim of this paper is to provide the technical basis for intraoperative HSI data acquisition on patients in neurosurgery. It will be shown that it is possible to connect a commercially available high-resolution HSI camera technology (TIVITA Tissue Camera, Diaspective Vision GmbH, Germany) due to its small size as well as an associated light source to a surgical microscope (OPMI Pico, Carl Zeiss Meditec AG, Germany) (Figure 1). The HSI setup embedded in the routine workflow consists of six components: patient with trepanned skull, surgical microscope, light source, HSI camera, laptop with data acquisition, processing and visualization software, and clinical staff. Each of the mentioned components has its own individual drawbacks and limitations. To evaluate patient data, it requires detailed knowledge of the properties of the entire hardware system, an exact dark and white calibration, and the hardware-based pre-processing. In the following chapters, an example for a clinical intraoperative acquired hyperspectral data cube from a brain tumour resection will be shown. We concentrate on two components: characterization of the HSI system, partly described by calculation of the signalto-noise ratio (SNR) of image data and characteristics of light sources. The image quality and a possible evaluation with a machine learning algorithm are shown exemplarily.

\section{Materials and methods}

\section{Clinical workflow and setup for hyperspectral imaging process}

As shown in Figure 2, optical imaging in neurosurgery always consists of three phases: preoperative and intraoperative image data acquisition and intraoperative/postoperative image data evaluation. Preoperative data and the possibility of data acquisition during a brain tumour resection in combination with neuronavigation offer surgeons significant assistance. Our working group consisting of surgeons and engineers, developed a workflow for HSI data acquisition that makes it possible to bring HSI into the clinical routine for brain tumour resection without additional devices except the HSI camera. The HSI camera is connected with a conventional surgical microscope by a C-mount thread. During interventional preparation process, the camera is coupled to the same beam path of the microscope-like illumination and the ocular for the surgeon. We use 60 and $400 \mathrm{~mm}$ fixed focal length lenses, which belong to the microscope. The focus is adjusted to the patient's brain surface by changing the distance between brain tissue and lens of the $400 \mathrm{~mm}$ objective by the surgeon. During preparing the operating room, surgical nurse coats surgical microscope including HSI camera with a sterile cover. Covering a microscope is routinely performed by medical staff and is not an additional burden or disturbance. As shown in Figure 2 (centre right), microscope, light source, and HSI camera can be considered as one device-related unit. So the camera is not perceived as additional equipment and does not restrict neither the physician nor the clinical staff. The surgeon commonly continues the workflow for brain tumour resection by positioning the surgical microscope for opening the skull and exposing the cortex.

Light is emitted by the light source via a fibre optic into the beam path of the surgical microscope through a lens to the cortex. Fraction of reflected light that passes the same lens like the emitted light into the beam path is projected through the slit of the pushbroom HSI camera onto the sensor. After hyperscanning of the trepanned skull and hardware-based raw data pre-processing (see below), hyperspectral image data cube is transferred to data processing and visualization software at a laptop. The software provides information about location and boundaries of the tumour. After intraoperative evaluation of visualized spatial and spectral data, the surgeon can proceed with brain tumour resection. In postoperative image evaluation phase of this workflow, all acquired hyperspectral data from the intraoperative phase are matched and evaluated with established medical image processing methods. The identical workflow of IOI and HSI as well as the possibility to use different cameras at the same time with one surgical microscope gives the opportunity to measure IOI and HSI synchronously. This provides data fusion to get further information from a combination of all imaging methods.

\section{Characteristics of the lighting}

In a hyperspectral measuring setup in clinical surrounding, used light sources should have the following properties: high intensity in a spectral range between 500 and 1,000 nm, nearly homogeneous spectral characteristics of lighting, and no dangerous warming of the brain tissue to avoid any damage to the human cells. If the temperature of illuminated brain tissue rises up to 41 to $44^{\circ} \mathrm{C}$ in animal models, the central nervous and neural system will show verifiable cell 


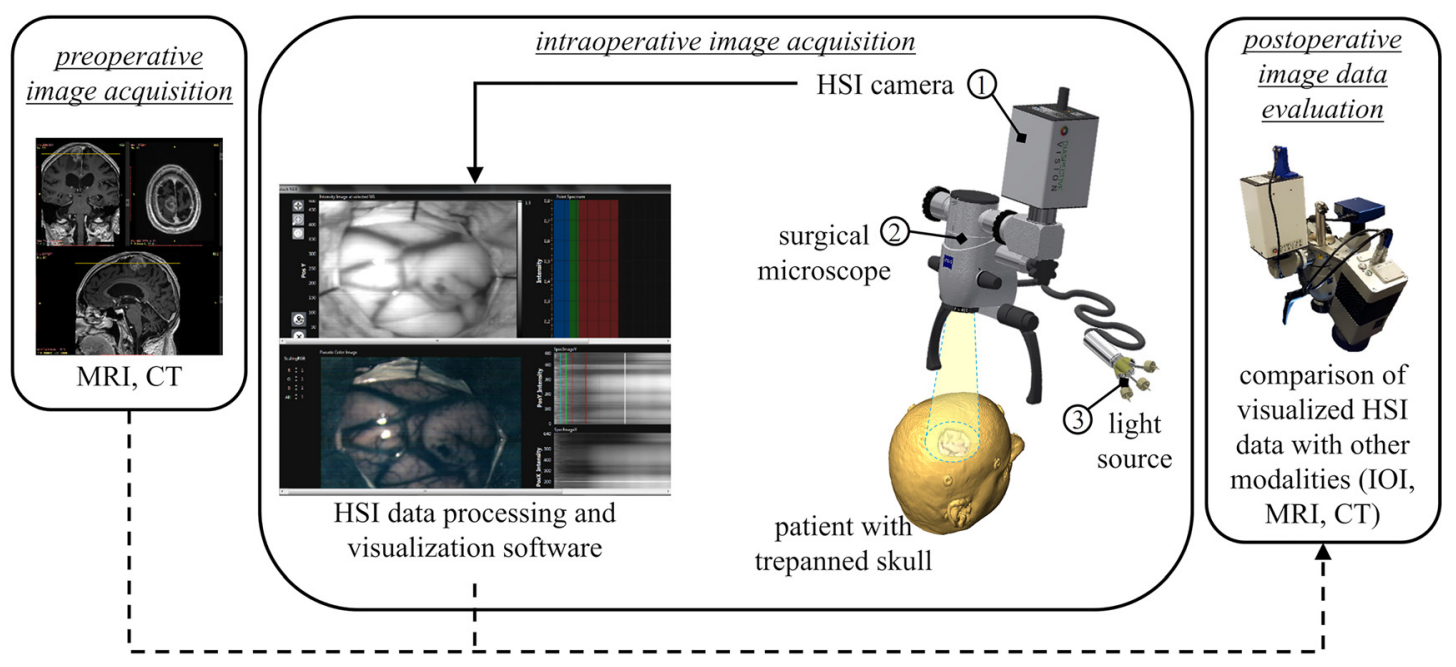

Figure 2: Workflow for optical imaging in neurosurgical research with hyperspectral data acquisition during brain tumour resection. In neurosurgery, the imaging process is divided into three different phases: preoperative (left) and intraoperative image data acquisition (middle), and intraoperative/postoperative image data evaluation (right). The dashed arrows symbolize the navigated data flow between these three phases. HSI camera (1), surgical microscope (2), and light source (3) form a device-related unit. This unit is covered with a conventional sterile microscope cover before the resection starts. During the intervention, the surgeon is positioning this unit over the trepanned skull for hyperspectral image data acquisition. After scanning the tissue and pre-processing raw data, the recorded data cube is transferred to the data processing and visualization software (solid arrow). In postoperative image data evaluation phase, the recorded hyperspectral data is compared with other image data from established medical image processing methods and subsequently evaluated.

damages [20]. In addition to this study, the maximum optical power of the light source has to be $2.27 \mathrm{~W} / \mathrm{cm}^{3}$ to reach a warming of $0.5^{\circ} \mathrm{C}$ of the brain tissue of a rat [21].

For our clinical measuring setup, lighting has to focus onto the fibre optics of the microscope in order to couple the light into the beam path of the surgical microscope. We used halogen lamps with a quasicontinuous spectrum between 200 and 2,000 nm. Xenon or LED lamps show high peaks in their spectral characteristics; therefore, we refused the application. The quantum efficiency of the HSI camera (TIVITA Tissue Camera) has the highest sensitivity at $550 \mathrm{~nm}$ and decreases up to $1,000 \mathrm{~nm}$. This effect can be compensated by high radiation intensity in near infrared (NIR) range of halogen lamp.

In this work, 18 different halogen lamps (Table 1) were examined. They differ in design, and orientation of the spiral-wound filament, and composition of the gas, and in reflector material. In addition to the design, the shape of the spectral characteristic and the wavelengthdependent intensity, which are influenced by the type of reflector and the composition of the gas, are important for this work.

All of the 18 halogen lamps were operated with manufacturer advertised nominal power and light (in a range between 200 and $1,000 \mathrm{~nm}$ ) were measured by direct irradiation with a spectrometer (USB2000+VIS-NIR-ES, beam path: QR400-7-VIS-BX, Ocean Optics, Inc., USA). The spectrometer has a spectral resolution of $1.5 \mathrm{~nm}$ and covers a wavelength range between 350 and $1,000 \mathrm{~nm}$. The SNR of this gauge is 250:1 and it has a dynamic range of 1,300:1 single acquisition with a single dark spectrum of 50 counts root mean square (RMS). The system has a dynamic range of $2 \times 10^{8}$. In this experiment, the spectral range is limited from 500 to $1,000 \mathrm{~nm}$. The experiments were performed by averaging 10 scans and by setting a variable integration time per scan. For every lamp, an individual exposure time was used. For this reason, results and discussion deal only with the shape of spectral characteristics and not with their absolute values.

\section{Characteristics of the hyperspectral imaging camera}

Before starting data acquisition during brain tumour resection in neurosurgical environment, the new HSI system should be thoroughly examined with regard of the quality of its technical parameters and the associated technology should be adapted to the clinical environment. Only after a complete optimization of the technical parameters for the intraoperative setup at a laboratory workstation under comparable boundary conditions, it will be possible to work intraoperatively with the entire system on the patient to give relevant statements for the physician.

The used laboratory setup with special subsystems simplified for research is similar to most of the state-of-the-art systems mentioned in the introduction because it offers the possibility of an almost spatially homogeneous illumination of the field of view, a high irradiance and an individual choice of camera lens. Sensitivity within the camera wavelength range and spatial resolution are to be identified. SNR is required as high as possible to interpret measurements during intraoperative recordings. To characterize the influence of the measuring setup on data quality, experimental and intraoperative setups are compared by calculating SNR at nine points in spatial dimensions for each wavelength between 500 and $995 \mathrm{~nm}$.

TIVITA tissue camera: Main item of the HSI camera is a CMOS sensor (AR0130CS, On Semiconductor Corp., USA) with a resolution of $1,280 \times 960$ pixel. Capturing a sensor image, the dimensions $\mathrm{y}$ and $\lambda$ are recorded: $\mathrm{y}$ is the spatial dimension along the entrance slit, and $\lambda$ is the wavelength information. The used HSI camera system TIVITA Tissue Camera is a line-scan camera with a pushbroom scanner. The pushbroom technology is based on scanning object's surface in columns during a movable slit. HSI 
Table 1: Technical parameters of the tested lamps.

\begin{tabular}{|c|c|c|c|c|}
\hline nr. & Company, name & power & voltage & reflector \\
\hline 1 & OSRAM Ministar 50050 WFL & $50 \mathrm{~W}$ & $12 \mathrm{~V}$ & aluminium \\
\hline 2 & OSRAM Ministar $50020 \mathrm{WFL}$ & $20 \mathrm{~W}$ & $12 \mathrm{~V}$ & aluminium \\
\hline 3 & OSRAM Bellaphot 64605 & 50 & $8 \mathrm{~V}$ & dichroic \\
\hline 4 & OSRAM Xenophot 64634 HLX EFR & $150 \mathrm{~W}$ & $15 \mathrm{~V}$ & dichroic \\
\hline 5 & OSRAM Halospot 4841910 & $10 \mathrm{~W}$ & $12 \mathrm{~V}$ & aluminium \\
\hline 6 & OSRAM Halostar 64405 & $5 \mathrm{~W}$ & $12 \mathrm{~V}$ & none \\
\hline 7 & OSRAM DecoStar 4186151 ALU WFL & $50 \mathrm{~W}$ & $12 \mathrm{~V}$ & aluminium \\
\hline 8 & OSRAM DecoStar 41894 WFL & $50 \mathrm{~W}$ & $12 \mathrm{~V}$ & dichroic \\
\hline 9 & OSRAM DecoStar 44892 WFL & $35 \mathrm{~W}$ & $12 \mathrm{~V}$ & aluminium \\
\hline 10 & OSRAM DecoStar 4186151 ALU WFL & $20 \mathrm{~W}$ & $12 \mathrm{~V}$ & aluminium \\
\hline 11 & Narva & $150 \mathrm{~W}$ & $12 \mathrm{~V}$ & none \\
\hline 12 & Narva & $100 \mathrm{~W}$ & $12 \mathrm{~V}$ & none \\
\hline 13 & Narva & $75 \mathrm{~W}$ & $12 \mathrm{~V}$ & none \\
\hline 14 & Philips & $50 \mathrm{~W}$ & $12 \mathrm{~V}$ & aluminium \\
\hline 15 & Sygonix $20367 \mathrm{Y}$ & $35 \mathrm{~W}$ & $12 \mathrm{~V}$ & aluminium \\
\hline 16 & Sygonix 28929V & $35 \mathrm{~W}$ & $12 \mathrm{~V}$ & aluminium \\
\hline 17 & Heitronic & $10 \mathrm{~W}$ & $12 \mathrm{~V}$ & none \\
\hline 18 & Nice Price & $20 \mathrm{~W}$ & $12 \mathrm{~V}$ & none \\
\hline
\end{tabular}

camera captures $640 \mathrm{y}-\lambda$-images along the spatial $\mathrm{x}$-axis and merges them into a hyperspectral $\mathrm{x}-\mathrm{y}-\lambda$-data cube $[11,22]$.

TIVITA Suite: The software TIVITA Suite (Version 0.6.1.4, Diaspective Vision GmbH, Germany) was used for first experimental evaluations. This software reduces the hyperspectral data cube to $640 \mathrm{px}$ in scanning direction $\mathrm{x}, 480 \mathrm{px}$ in slit direction $\mathrm{y}$, and to 100 wavelengths with a $5 \mathrm{~nm}$ resolution in wavelength range $\lambda$. Previous to each experiment, dark cube $\left(I_{\mathrm{Dx}, \mathrm{y}, \lambda}\right)$ and white reference cube $\left(I_{0 \mathrm{x}, \mathrm{y}, \lambda}\right)$ from lighting on a white reference area have to be recorded and stored by the software. Each dark cube was converted by averaging the measured intensities in black environment per pixel over the wavelengths to a two-dimensional image, the dark pattern $\left(I_{\mathrm{Dx}, \mathrm{y}}\right)$. The recorded raw intensity values $\left(I_{\mathrm{RAWx}, \mathrm{y}, \lambda}\right)$ are calculated according to Formula (1) [22] to a pre-processed intensity data cube $\left(I_{\mathrm{x}, \mathrm{y}, \lambda}\right)$.

$$
I_{x, y, \lambda}=\frac{I_{\mathrm{RAW}_{x, y, \lambda}}-I_{\mathrm{B}_{x}}-I_{\mathrm{D}_{x, y}}}{I_{0_{x, y, \lambda}}-I_{\mathrm{B}_{\mathrm{x}}}-I_{\mathrm{D}_{x, y}}} .
$$

To compensate thermally generated voltages, black level $\left(I_{\mathrm{Bx}}\right)$ is applied as image parameter and is adjusted by the camera sensor with each frame. This parameter is calculated from the optically black, light-insensitive pixels on the sensor and variable along the $\mathrm{x}$-axis within the recording of a data cube [22, 23].

SNR of the HSI camera images: For all subsequent experiments, a $45 \mathrm{~min}$ warm-up of the sensor and a $30 \mathrm{~min}$ burn-in time of the light sources were observed. Two setups were compared: Laboratory measuring setup "A" (see Figure 3A) contained a light source (6) which includes six 20 W halogen lamps (lamp 10, Table 1, OSRAM GmbH,
Germany), an optical lens (7) with $12 \mathrm{~mm}$ focal length, HSI camera (1), and a customized camera carriage setup (5). The clinical setup "B" (Figure 3B) consists of three $50 \mathrm{~W}$ halogen lamps (lamp 1, Table 1, OSRAM GmbH, Germany) (3), two optical lenses (OPMI Pico, Carl Zeiss Meditec AG, Germany) with a focal length of $400 \mathrm{~mm}$ (8a) and $60 \mathrm{~mm}$ (8b), HSI camera (1), a surgical microscope (S100 OPMI Pico, Carl Zeiss Meditec AG, Germany) (2) and a fibre optics (Carl Zeiss Meditec Vertriebsgesellschaft mbH, Germany) (4). For both setups, the distance between optical lens and white standard (Zenith Polymer® Diffusers, SphereOptics GmbH, Germany) (9) or respectively a photographic grey card (JJC GC-1, Colour Balance Card, JJC Photography Equipment Co., Ltd., China) (9) was adjusted to $400 \mathrm{~mm}$.

Due to different light sources (3 and 6) and the use of fibre optics (4) in setup B, different illumination areas were produced on white standard / grey card (9) (see Figure 3). In setup A, illuminated area is larger than $250 \times 250 \mathrm{~mm}^{2}$ and, in setup B, illuminated area is $140 \mathrm{~mm}$ in diameter. The illuminance $\left(E_{v}\right)$ averaged over the surface of setup $A$ is 10 times higher than the illuminance $\left(E_{v}\right)$ averaged over the surface of setup B. With both setups, it is not possible to illuminate the measurement object spatially homogeneous. That's why, there were differences in the software parameter settings of the setups. In setup A, an exposure time of $25 \mathrm{~ms}$, an analogue gain of one, digital gain of 32, and an aperture of 1.4 were fixed, and in setup B an exposure time of $80 \mathrm{~ms}$, analogue gain of eight, and digital gain of 32 are used at the same aperture like setup A. For each setup, after saving $\mathrm{I}_{\mathrm{Dx}, \mathrm{y}}$ and $\mathrm{I}_{\mathrm{Ox}, \mathrm{y}, \lambda}$, one hyperspectral image of white standard (noise cube) and 40 hyperspectral image cubes of grey card (signal cubes) were recorded. In order to calculate the SNR, the noise cube and the signal cubes have to be converted into the absorbance intensity data cube $I_{A x, y, \lambda}$ by Formula (2): 


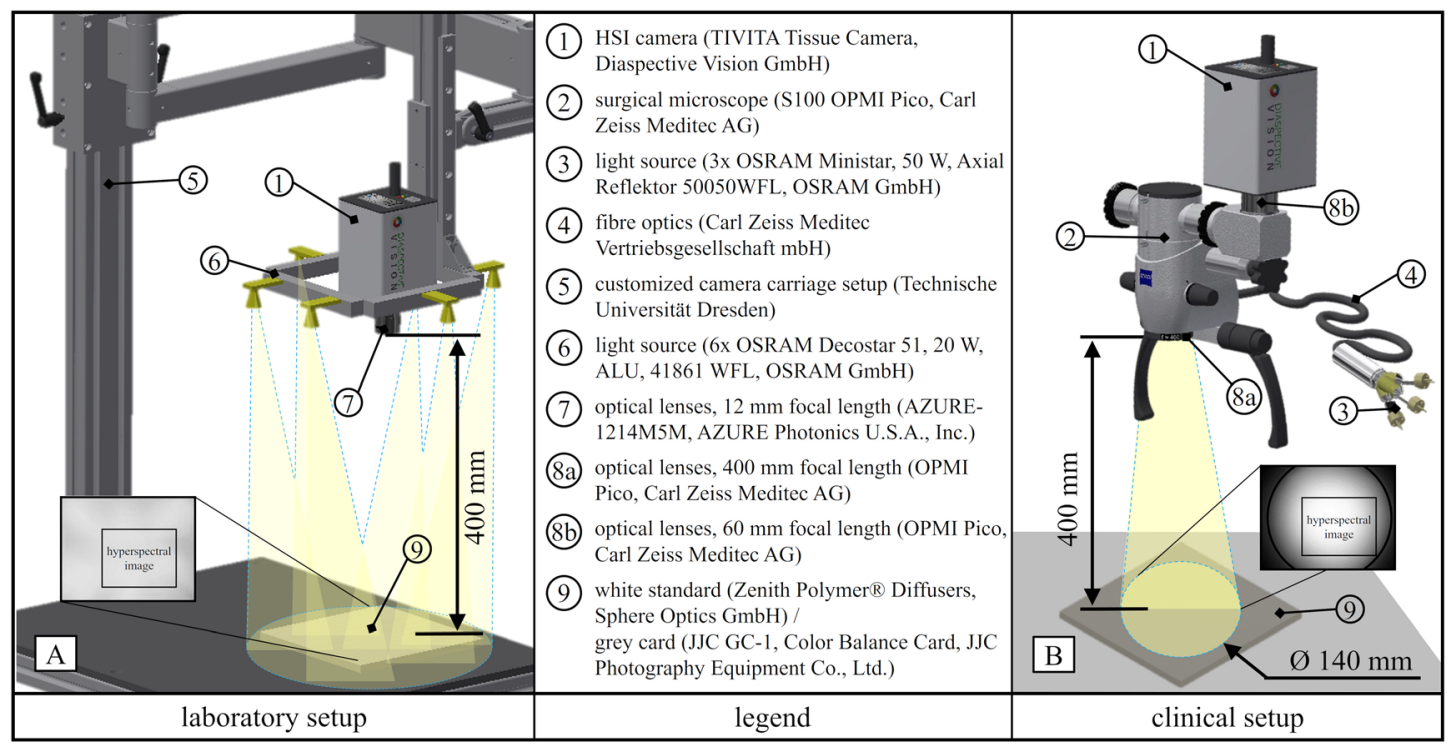

Figure 3: Two measuring setups: laboratory setup (A) and clinical setup (B). The main components of setup A are: HSI camera (1), customized camera carriage setup (5), light source (6), optical lenses (7) and a white standard/grey card (9). The main components of setup B are: HSI camera (1), surgical microscope (2), light source (3), fibre optics (4), optical lenses ( 8 a and 8b) and a white standard/grey card (9). The distance between optical lenses and the white standard/grey card is $400 \mathrm{~mm}$ in both setups. Setup A: The illuminated area is larger than $250 \mathrm{~mm}$ diameter. Setup B: The illuminated area is $140 \mathrm{~mm}$ in diameter.

$$
I_{A_{x, y, \lambda}}=-\lg I_{x, y, \lambda}
$$

After that, all of the 40 signal cubes were averaged pixel by pixel for setups A and B. Signal and noise power were calculated for each data point pixel by pixel and depending on the wavelength. SNR was calculated by Formula (3), where $P$ is the average power:

$$
\operatorname{SNR}(\lambda)=10 * \lg \frac{P_{\text {signal }}(\lambda)}{P_{\text {noise }}(\lambda)}
$$

\section{Spectral data acquisition and raw data pre-processing in neurosurgery}

In order to show the possibility to record intraoperative HSI data using the workflow and setup described below, an intraoperative image data cube was recorded. Exposure time of $80 \mathrm{~ms}$, analogue gain of eight, and digital gain of 32 for the data acquisition were set like setup B (Figure 3). For the experiment, a low-grade oligodendroglioma (WHO III) from a 39-years-old male patient was analysed after tumour resection.

\section{Spectral data evaluation}

The development of automatic segmentation tools does not fall within the scope of this paper. However, an exemplary evaluation of the obtained hyperspectral data shall be performed to visualize the measured raw data. For that a machine learning algorithm is used to differentiate relevant regions in the image. Although the application of training and test data to the same patient data set is questionable, a part of the tumour and brain pixels shall be used for learning and the remaining areas of the trepanation for the application of the classifier.
As can be seen from Figure 7, these regions are background (cranial bone, dura mater, medical suture material, and surgical drapes), vessels, brain tissue, tumour tissue, and specular reflections. Specular reflections drive the camera detector elements into saturation, which deletes all information about these pixels. Due to their broadband high intensities, specular reflections can be identified and segmented threshold-based. The background is determined by manual ROI selection. Since manual selection is slow and might not always exactly match the trepanation border, discoid dilation with five pixel radius expands the background mask.

Vessel detection is performed by two approaches, because small and big vessels affect acquired spectra varyingly strong. Formula 4 shows the detection of small vessels via threshold $s_{v}$. Haemoglobin exhibits high absorbance at the wavelength $\lambda=590 \mathrm{~nm}$. Hence, big distances to the maximum intensity in the $5 \times 5$-neighbourhood around the small vessel pixels are expected. Big vessels can be extracted by spectral angle mapping. At first, the operator chooses a reference pixel from the tissue area. After that, the angle between the spectrum of each pixel and the reference spectrum of the chosen tissue pixel is calculated. Therefore, a pixel spectrum is understood as a vector. The spectra of big vessels are expected to be dissimilar to brain and tumour spectra due to the dominant absorption of haemoglobin. Finally, an angular threshold splits the big vessels from surrounding tissue. The masks for small and big vessels are merged by a Boolean logical OR operator.

$$
\frac{\max _{x, y \in \text { Neighbourhood }}\left(I_{x, y, \lambda=590 \mathrm{~nm}}\right)-I_{x, y, \lambda=590 \mathrm{~nm}}}{I_{x, y, \lambda=590 \mathrm{~nm}}}>S_{V}
$$

For the distinction of brain and tumour tissue, supervised learning algorithms such as the Random Forest (RF) classifier are suitable. The selection of required reference areas follows the presumed tumour border (Figure 7). This border was highlighted by an 
experienced neurosurgical expert under the use of preoperative MRI data. Because of the infiltrating tumour growth, reference areas were chosen with big distance to the presumed tumour border (Figure 7). The reference area covers 26,915 brain and 11,759 tumour pixel spectra. For noise reduction, the hypercube is filtered by the HyRes algorithm before it reaches the RF classifier [24, 25]. Furthermore, specular reflections, background, and vessels are removed from the spectral image data. The RF classifier with 300 decision trees at optimum is trained and tested on the reference area by 5 -fold stratified cross validation.

At last, the RF classifier is trained on the whole cleaned reference area and predicts the class labels (brain or tumour) for all remaining pixels in the image. This information is merged with the masks for specular reflections, background, and vessels. The resulting classification map is layered over an anatomical image for the surgeon's orientation (Figure 7).

\section{Results}

\section{Spectral characteristics of the lighting}

Figure 4 shows spectral characteristics of 18 different halogen lamps with aluminium reflector (red and orange),

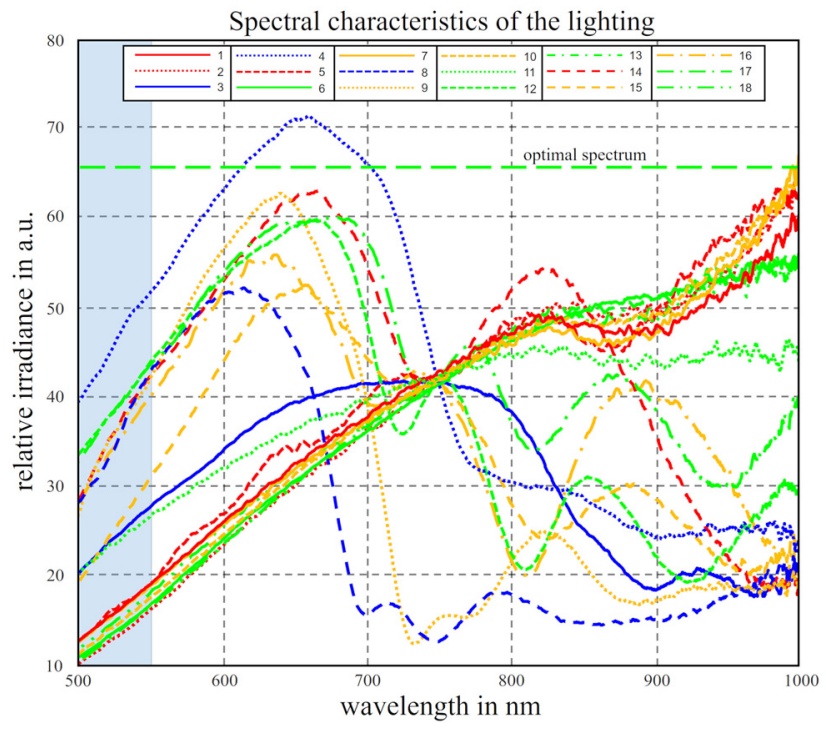

Figure 4: Spectral characteristics of 18 different halogen lamps (Table 1). Aluminium reflector (red and orange): Osram Ministar 50050 WFL (50 W, 12 V) (1), Osram Ministar 50020 WFL (20 W, 12 V) (2), Osram Halospot 4841910 (10 W, 12 V) (5), Osram DecoStar 41861 ALU WFL (50 W, 12 V) (7), Osram DecoStar 44892 WFL (35 W, 12 V) (9), Osram DecoStar 4186151 ALU WFL (20 W, 12 V) (10), Philips (50 W, 12 V) (14), Sygonix $20367 Y$ (35 W, 12 V) (15) and Sygonix 28929 V $(35 \mathrm{~W}, 12 \mathrm{~V})(16)$. Without reflector (green): Osram Halostar 64405 (5 W, 12 V) (6), Narva (150 W, 12 V) (11), Narva (100 W, 12 V) (12), Narva $(75 \mathrm{~W}, 12 \mathrm{~V})$ (13), Heitronic (10 W, 12 V) (17) and Nice Price (20 W, $12 \mathrm{~V})$ (18). Dichroic reflector (blue): Osram Bellaphot 64605 (50 W, 8 V) (3), Osram Xenophot 64634 HLX EFR (150 W, 15 V) (4) and Osram DecoStar 41894 WFL (50 W, 12 V) (8).
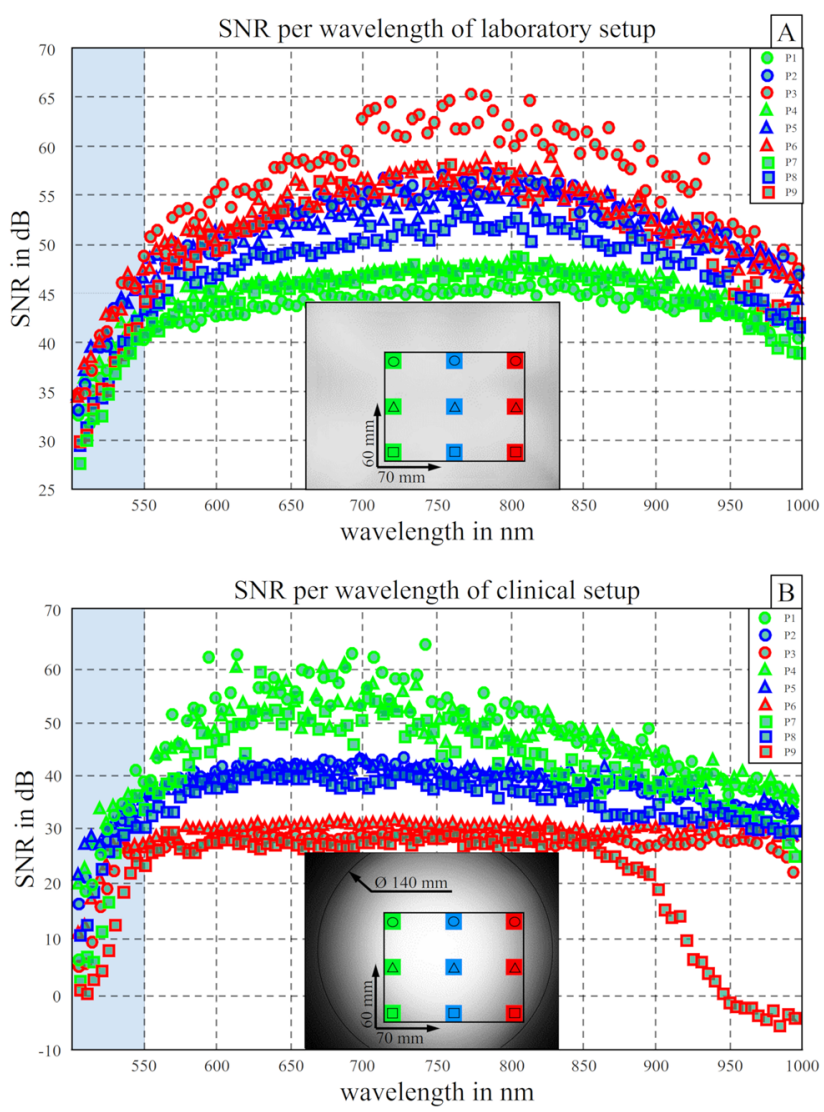

Figure 5: Averaged SNR calculated over $10 \times 10$ pixels per wavelength and area of setups $A$ and $B$. The light blue transparent marked area is characterized by a sharp fall in the SNR values. The exact positions of the averaged areas: $P_{1}(1-10 ; 471-480), P_{2}$ (316325 ; 471-480), $P_{3}(631-640 ; 471-480), P_{4}(1-10 ; 236-245), P_{5}(316-$ $325 ; 236-245), P_{6}(631-640 ; 236-245), P_{7}(1-10 ; 1-10), P_{8}$ (316$325 ; 1-10)$ und $P_{9}(631-640 ; 1-10)$. The same colour shows areas the same $y$-dimension (slit direction) and the same symbol shows areas in the same $x$-dimension (scanning direction).

dichroic reflector (blue), and without any kind of reflector (green). Depending on filler gas and material of the filament, spectra show different characteristic bands in the range between 500 and 1,000 $\mathrm{nm}$. Different types of lines help to distinguish different spectra from each other. The light blue transparent area represents the wavelength range with a low SNR of the HSI camera (see Figure 5). On the y-axis, the relative irradiance is applied to make the different data comparable. The wavelengths in $\mathrm{nm}$ are plotted on the $x$-axis. Most interesting light sources for the application of hyperspectral imaging in neurosurgery, shown in Figure 4, are lights with a constant rise of the irradiance between 500 and 1,000 $\mathrm{nm}$. In addition, lighting should not have any local extremes in their spectrum. Except the small plateau between 800 and $900 \mathrm{~nm}$ spectral, characteristics of light sources 1 (Table 1, Figure 4) and 10 (Table 1, Figure 4) represent a relatively constant 

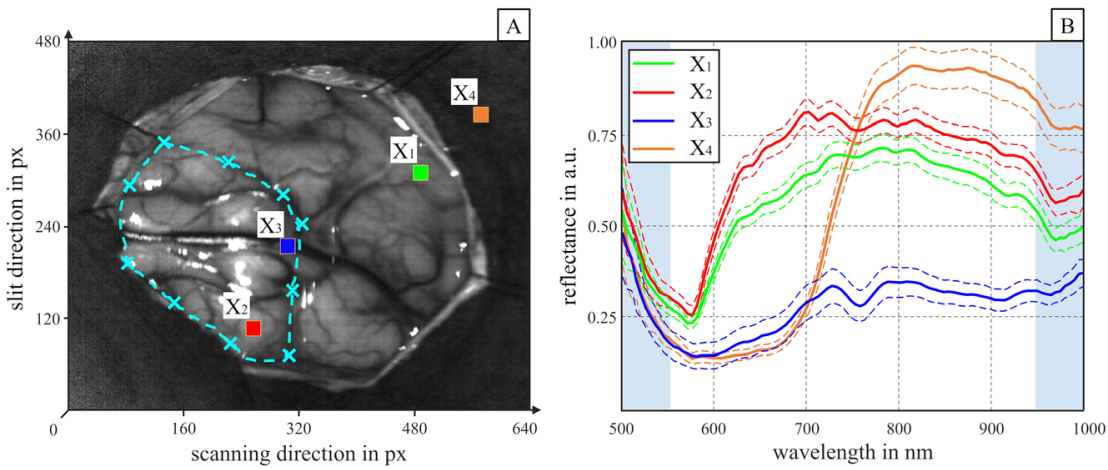

Figure 6: First intraoperative HSI image data acquisition during brain tumour resection in neurosurgery of patient ID008 and spectra from exemplary regions of the hypercube. (A) Grey-scale image of the $600 \mathrm{~nm}$ band from the hypercube is shown. Four coloured squares mark exemplarily $15 \times 15$-neighbourhood pixels for different areas. Cyan dashed line marks the presumed tumour border that was highlighted by an experienced neurosurgeon under the use of MRI data. (B) Spectra from exemplary regions shown in panel $(A)$ can be seen. The solid line indicates the mean values of the

225 neighbourhood pixels. The dashed lines represent the standard deviation values proceeding from the mean values. The spectral signatures vary strongly between the four areas. The transparently blue marked area represents the range with bad SNR according to the chapter above.

increase compared to others like lamp number 11 (Table 1, Figure 4) or lamp number 4 (Table 1, Figure 4). The spectral characteristics of lamp number 4 (Table 1) has a maximum at $650 \mathrm{~nm}$. In wavelength range between 700 and $800 \mathrm{~nm}$, values drop rapidly. Data of the light source 11 (Table 1) rises continuously up to $750 \mathrm{~nm}$. After this wavelength, they remain constant up to $1,000 \mathrm{~nm}$.

\section{SNR of the HSI camera}

Figure 5 shows the SNR in different areas of a hyperspectral image data cube in dependence on the wavelength. In the outer larger diagram, the wavelengths in $\mathrm{nm}$ are plotted against the SNR in $\mathrm{dB}$. The small figure (Figure 5 bottom middle) represents a $2 \mathrm{D} x$-y-image consisting of the position of important areas for SNR calculation and lighting situation on the test object. Averaged areas with the same symbol lying in the same $\mathrm{x}$ direction (scanning direction) and averaged areas with the same colour lying in the same $y$ direction (slit direction). The area size is $10 \times 10$ pixels. The illumination of the surface is caused by the different two light sources (see Figures 3A, B, and 6). With both it is not possible to illuminate the measurement object spatially homogeneous. The laboratory setup does not illuminate the surface spatially homogeneously (Figure 5A). It varies between 11,100 and 8,700 lux. The illuminance of the surface from the medical setup is darkened evenly from the centre (3,900 lux) to the edge (Figure 5B, red square, 2,200 lux). In the spectral range between 500 and $550 \mathrm{~nm}$ (light blue transparent marked range), the SNR falls sharply. In further experiments of this work, this wavelength area will not be considered because of the low SNR. Figure 5A represents the SNR distribution of the laboratory setup. For all nine areas, SNR in the wavelength range between 550 and
$970 \mathrm{~nm}$ was over $40 \mathrm{~dB}$. In spectral range from 970 to $1,000 \mathrm{~nm}$, the SNR of all areas decreases gradually, but does not reach $40 \mathrm{~dB}$. Most of the data from blue (P2; P5; P8) and red (P3; P6; P9) areas show SNR values over $50 \mathrm{~dB}$. The SNR values of area $\mathrm{P} 3$ in the wavelength range from 550 to $960 \mathrm{~nm}$ and 620 to $900 \mathrm{~nm}$ were higher $50 \mathrm{~dB}$ and higher $55 \mathrm{~dB}$ respectively. The SNR curves of three green areas $(\mathrm{P} 1$, P4 and P7) remain flat and show values between 40 and $50 \mathrm{~dB}$.

Figure 5B, SNR distribution of the clinical setup is shown. For all blue and green areas, the SNR is over $30 \mathrm{~dB}$ between 550 and $970 \mathrm{~nm}$. In this wavelength range, the SNR of red areas is between 25 and $30 \mathrm{~dB}$, except area P9. The green areas have a SNR of more than $45 \mathrm{~dB}$ between 575 and $850 \mathrm{~nm}$. In both measuring setups, the SNR in regions higher than $50 \mathrm{~dB}$ show more fluctuation than lower $\mathrm{dB}$ regions. Starting at $850 \mathrm{~nm}$, the SNR values of area P9 show a sharp decrease down to $-5 \mathrm{~dB}$. Figure $5 \mathrm{~B}$ shows a light spot with $140 \mathrm{~mm}$ diameter in the middle of the measuring object with a dark surrounding. The recorded hyperspectral image is at the lower right edge of the illuminated circle.

\section{Data acquisition and raw data pre- processing during neurosurgical operation}

A total of 18 patient data sets have already been recorded with the existing intraoperative HSI system. In order to illustrate the technical approach, all data presented in this paper are taken from patient ID008. Further analysis of all available patient data sets will be published in the next paper.

Figure $6 \mathrm{~A}$ represents a grey-scale image calculated from HSI image data. It has a resolution of $640 \times 480$ pixel. 
The scanning direction is plotted on the $\mathrm{x}$ axis and the slit direction on the y axis. Red, green, orange and blue marked areas consist of a $15 \times 15$ field of pixels. Reflectance values averaged to plotted colour coded areas have a resolution of $5 \mathrm{~nm}$ and they are represented in Figure 6B. They are exemplarily for all pixels of the grey-scale false-colour image. The trepanned skull is shown in the brighter central area of Figure 6A. Outer dark areas are the sterile surgical drapes. The black dark lines are the medical suture material used to stretch and hold dura mater and surgical drapes. The recognizable vascular structure is distributed over the cortex. White dots scattered over the cortex are light reflections. At these points, the reflection is nearby $100 \%$. The green mark (X1) exemplarily represents the spectra of the exposed healthy cortex, the red mark (X2) the exposed malignant cortex, the blue mark (X3) the exposed cortex in the middle of the trepanation (vessel or sulci) and the orange mark (X4) a surgical drape. The light blue transparent marked area represents the wavelength range (500-550 nm) with a low SNR (see above). Each averaged surface shows different spectral characteristics. In future, it will be the task to assign different characteristics to different tissue types and to examine properties on pixel basis.

\section{Spectral data evaluation}

Figure 7 shows the extracted classification map. Specular reflections are covered accurately. The segmentation of big horizontal vessel works perfectly, but small vessels are just partially detected. Outside the reference area brain tissue dominates. Tumour regions appear as intrinsically connected spots. The cleaned reference area covers 22,157 brain and 6,596 tumour pixel spectra. In the 5 -fold stratified cross validation scenario, the RF classifier reaches an accuracy of $99.1 \%$. Sensitivity and specificity both exceed $98 \%$.

\section{Discussion}

Figures 6 and 7 show the possibility to integrate an HSI measurement setup into a brain tumour resection workflow and the functionality of the hyperspectral imaging process.

Because of the centre of the optical system's lens is not the centre of the image, the red areas in Figure $5 \mathrm{~A}$ and the green areas in Figure 5B have the highest SNR values. The HSI camera can be rotated on the surgical microscope, it can be led to a change of the scanning direction. This is the reason why highest and lowest SNR values in Figure 5A, B

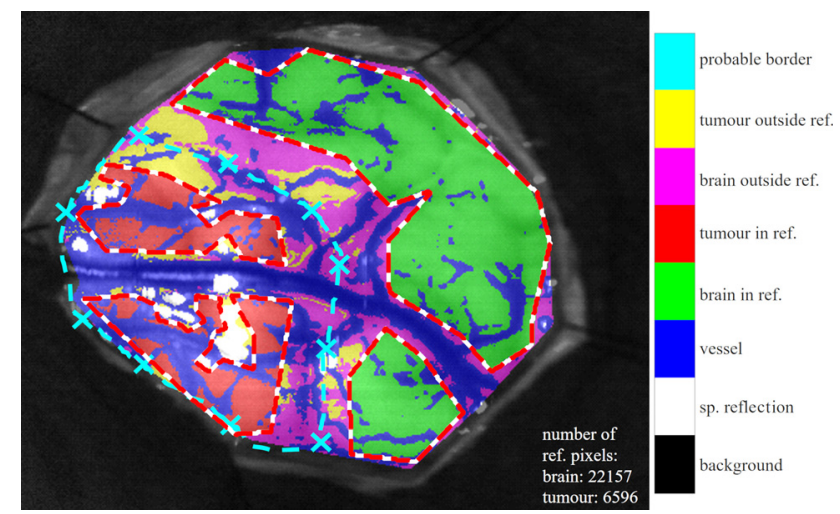

Figure 7: Classification map with the presumed tumour border (cyan dashed) and borders of the reference areas (red-white dashed, green and red pixel) over the grey-scale image of the $625 \mathrm{~nm}$ wavelength of patient ID008. The tumour border of patient was intraoperatively highlighted by an experienced neurosurgeon under the use of MRI data. Due to infiltrating tumour growth, the reference areas keep distance from the border. Next to brain and tumour tissue, the image contains the classes specular reflections, background and vessels. Specular reflections and the big horizontal vessel are covered accurately. Small vessels are just partially detected. Mainly due to the vessel segmentation, the cleaned reference areas contain fewer pixels than the original reference ROIs. The RF classifier for the brain-tumour distinction was trained on the whole cleaned reference area. Outside the reference, brain pixels dominate. Tumour regions appear as intrinsically connected spots. Both groups cross the tumour border.

have not the same colour. Areas at the edge of the lens have a lower SNR than areas in central of the optical lens because of vignetting and distortion. Another reason is the low sensor sensitivity in combination with the lower illuminance. This results in more noise artefacts and thus reduces the SNR. The difference between clinical measuring setup and laboratory measuring setup can be visualized by different SNR characteristics of each averaged colour coded area. Laboratory measuring setup has a much higher SNR, which is caused by more powerful illumination among other factors. More powerful illumination results in a shorter exposure time and faster measurement. This is illustrated by the settings and recordings for setup A and B in Figure 3.

Laboratory setup A, 25 ms exposure time and an analogue gain of one were set. For clinical setup B (Figure 3), $80 \mathrm{~ms}$ exposure time and an analogue gain of eight had to be set. This resulted in a $50 \mathrm{~s} /$ datacube faster recording time and a higher SNR of setup A.

In conclusion from the results of the SNR measurements, wavelength range between 500 and $550 \mathrm{~nm}$ cannot be evaluated without errors of both systems, which is basically caused by camera-specific characteristics. In addition, SNR can be increased by changing the 
illumination ratios. The results show that the SNR achieves good values and the HSI data can thus be evaluated in both systems between 550 and $1,000 \mathrm{~nm}$. There is a limitation in the border area, which is clearly shown by the SNR results in the area of the red square in Figure 5B.

In this work, it was not possible to use the same exposure time of the spectrometer due to the different power values of the light source (see above). For this reason, only the shape of spectral characteristics is considered and not the absolute values of spectral intensity. The increasing noise of the spectral lines of the spectrometer is due to the quantum efficiency of the sensor and the spectral grating in this wavelength range. Whereas the strong drop of lamp 4 (Table 1) comes from the dichroic reflector. This coating reflects radiation below $650 \mathrm{~nm}$ and transmits radiation at higher wavelengths. As a result, heat radiation is absorbed and not delivered to the underlying measurement object. Higher power of the lamp itself does not simultaneously provide better spectral characteristics for HSI data acquisition. Important is the material of the reflector of the lamp or the lamp itself as well as a statement whether important spectral components are reflected or absorbed by the material of lamp/ the reflector of the lamp.

If the camera has a continuous high quantum efficiency in the higher wavelength range, illumination does not have to be a halogen emitter, which brings power into the tissue in the illuminated area because this could be increases the temperature of the tissue. However, if the quantum efficiency of the HSI camera decreases in the higher wavelength range, the illuminant should rather have high intensity in these areas.

That's why we used lamp 1 (Table 1) for clinical measuring setup and lamp 10 (Table 1) for laboratory setup. Lamp 1 (Table 1) has a small design, no dichroitic reflector and the reflector emits the light almost perpendicular to the front. For the HSI-system, this means higher illuminance in the field of view and an undistorted halogen spectrum. Whereas for the measuring setup in laboratory, geometrical design of the light source is not important. But it was important that the systems could be compared easily, as well as a high luminous efficiency and a homogeneous local illumination over a large area. Due to the fact that the spectral sensitivity of the HSI camera which is used in this work decreases in the higher wavelength range, the used halogen lamps can compensate this property by the typical spectral rise of a halogen lamp in that range.

This HSI system compared to the Helicoid system, the difference in size becomes immediately apparent.
The Helicoid system brings an additional volume of $2,000 \times 600 \times 1,100 \mathrm{~mm}^{3}$ whereas this system does not exceed the dimensions of the S100 OPMI Pico. The OPMI Pico is a standard operating microscope which is used even without HSI measurements. In contrast to the helicoid system, the microscope can be covered sterilely with a standard Zeiss surgical drape. Therefore, it can be used in the sterile area of the operating theatre without restrictions.

The basic design of both systems is relatively identical with the difference that components such as positioning camera, "Up \& Down"-system and mobile tripod are integrated in the surgical microscope of the described HSI-system. Among other things, these aspects make the HSI-system much easier to handle for medical staff compared to the Helicoid system. What has not yet been already tested is the combination of different evaluation algorithms (e.g. Support Vector Machine, Artificial Neural Networks, Random Forest, Principal Component Analysis), as in the case of the Helicoid project $[12,18]$.

In laboratory care can be taken by choice of optical lenses, which is possible with restrictions in medical environment because of conditions of the surgical microscope. In neurosurgery, further influences are added by a second optical lens which is not examined in this work. White spots in Figure 6A are caused by directly reflected light, involved by the vertical position of the light source above the brain. This effect cannot be avoided because brain is an anatomically uneven surface and incident light is scattered back to the cortex via the same beam path as the reflected light from the cortex. As described above, the construction of the illumination unit is important in the intraoperative setup. Although we used three times lamp 1 (Table 1) with a total power of $150 \mathrm{~W}$, the exposure time has to be set to highest level.

In order to improve the existing system, the abovementioned total reflections could be virtually eliminated by a filter or homogenized light. In addition, optics of microscope could be improved and focus of lens could be adjusted by diffusers. For clinical setup, only one small lamp without any intern reflector should be installed to fit all defined conditions.

The delineated algorithm (Chapter Material, patients, and methods: Spectral data evaluation) interactively generates classification maps as shown in Figure 7. High values for accuracy, sensitivity, and specificity indicate that the RF classifier correctly differentiates between brain and tumour spectra in the reference area. For further algorithmic investigation, more data sets are required. 


\section{Conclusions}

It is important to know the characteristics of all components of a brain tumour resection workflow in neurosurgery in order to take account to all possible influencing factors for evaluation of a new imaging method. In this first investigation, it could be shown that it is possible to integrate a commercial HSI system into the intraoperative neurosurgical workflow during tumour resection. Especially light source, tripod and objective lens of an intraoperative optical imaging setup have to be adapted to the characteristics of the HSI camera and to the field of application.

It could be shown that it is possible to perform HSI data acquisition in a wavelength range from 550 to $1,000 \mathrm{~nm}$ with a spatial image of $640 \times 480$ pixel, spectral resolution of $5 \mathrm{~nm}$ and an acquisition time of 1 min during routine brain tumour resection in neurosurgery (Figure 7) to extent the amount of information about optical tissue properties got by other IOI procedures.

Acknowledgments: The authors would like to thank Julian Fischer for his advice and experimental assistance.

Research funding: This work was financially supported by the European Social Fund (ESF).

Author contributions: All authors have accepted responsibility for the entire content of this manuscript and approved its submission.

Competing interests: Authors state no conflict of interest.

\section{References}

1. Stummer W, Reulen H-J, Meinel T, Pichlmeier U, Schumacher W, Tonn J-C, et al. Extent of resection and survival in glioblastoma multiforme: identification of and adjustment for bias. Neurosurgery 2008; 62: 564-76.

2. Senft C, Bink A, Franz K, Vatter H, Gasser T, Seifert V. Intraoperative MRI guidance and extent of resection in glioma surgery: a randomised, controlled trial. Lancet Oncol 2011; 12: 997-1003.

3. Tharin S, Golby A. Functional brain mapping and its applications to neurosurgery. Oper Neurosurg 2007;60(4 Suppl):185-202.

4. Sobottka SB, Meyer T, Kirsch M, Koch E, Steinmeier R, Morgenstern $\mathrm{U}$, et al. Intraoperative optical imaging of intrinsic signals: $\mathrm{a}$ reliable method for visualizing stimulated functional brain areas during surgery. J Neurosurg 2013; 119: 853-63.

5. Meyer T, Morgenstern U, Kirsch M, Schackert G, Sobottka SB. Intraoperative optical imaging of intrinsic signals for delineation of active functional brain areas. In: Nabavi A, Samii A, Fahlbusch R, editors. Visualization of the brain and its pathologies - technical and neurosurgical aspects; 2016. ISBN/EAN: 9783862475773. pp. 92-175.
6. Meyer T, Sobottka SB, Kirsch M, Schackert G, Steinmeier R, Koch E, et al. Intraoperative optical imaging of functional brain areas for improved image-guided surgery. Biomed Tech 2013; 58 : 225-36

7. Sobottka SB, Meyer T, Kirsch M, Koch E, Steinmeier R, Morgenstern $U$, et al. Evaluation of the clinical practicability of intraoperative optical imaging comparing three different camera setups. Biomed Tech 2013; 58: 237-48.

8. Oelschlägel M, Meyer T, Schackert G, Kirsch M, Sobottka SB, Morgenstern U. Intraoperative optical imaging of metabolic changes after direct cortical stimulation - a clinical tool for guidance during tumor resection?. Biomed Tech 2018; 63: 587-94.

9. Raab P, Pilatus U, Lanfermann H. Spektroskopie bei Hirntumoren. Radiologie up2date. 2008; 8: 239-55.

10. Holm E. Stoffwechel und Ernährung bei Tumorkrankheiten: Analysen und Empfehlungen, 15th ed. New York; 2007: vol. 2007, 41-58 pp.

11. Lu G, Fei B. Medical hyperspectral imaging: a review. J Biomed Opt 2014;19. 010901. https://doi.org/10.1117/1.JBO.19.1. 010901.

12. Fabelo H, Ortega S, Lazcano R, Madroñal D, M CG, Juárez E, et al. An intraoperative visualization system using hyperspectral imaging to aid in brain tumor delineation. Sensors 2018; 18 : 430.

13. Markgraf W, Feistel P, Thiele C, Malberg H. Algorithms for mapping kidney tissue oxygenation during normothermic machine perfusion using hyperspectral imaging. Biomed EngBiomed Te 2018; 63: 557-66.

14. Shapey J, Xie Y, Nabavi E, Bradford R, Saeed S, Ourselin S, et al. Intraoperative multispectral and hyperspectral label-free imaging: a systematic review of in vivo clinical studies. J Biophotonics 2019:12:e201800455.

15. Khan MJ, Khan HS, Yousaf A, Khurshid K, Abbas A. Modern trends in hyperspectral image analysis: a review. IEEE Access 2018; 6: 14118-29.

16. Ortega S, Fabelo H, lakovidis DK, Koulaouzidis A, Callico GM. Use of hyperspectral/multispectral imaging in gastroenterology. Shedding some-different-light into the dark. J Clin Med 2019; 8: 36.

17. Jansen-Winkeln B, Holfert N, Köhler H, Moulla Y, Takoh JP, Rabe $\mathrm{SM}$, et al. Determination of the transection margin during colorectal resection with hyperspectral imaging (HSI). Int J Colorectal Dis 2019; 34: 731-9.

18. Fabelo H, Ortega S, Kabwama S, Callico GM, Bulters D, Szolna A, et al. HELICoiD project: a new use of hyperspectral imaging for brain cancer detection in real-time during neurosurgical operations. In: Bannon DP, editor. International Society for Optics and Photonics; 2016: 986002p.book-chapter.

19. Fletcher JT, Kong SG. Principal component analysis for poultry tumor inspection using hyperspectral fluorescence imaging. In: Proceedings of the International Joint Conference on Neural Networks, IEEE; 2004:149-53pp.confproc.

20. Sharma HS, Hoopes PJ. Hyperthermia induced pathophysiology of the central nervous system. Int J Hyperthermia 2003;19: 325-54.

21. Ersen A, Abdo A, Sahin M. Temperature elevation profile inside the rat brain induced by a laser beam. J Biomed Opt 2014;19. 015009. https://doi.org/10.1117/1.jbo.19.1. 015009. 
22. Holmer A, Marotz J, Wahl P, Dau M, Kämmerer PW. Hyperspectral imaging in perfusion and wound diagnostics - methods and algorithms for the determination of tissue parameters. Biomed EngBiomed Te 2018; 63: 547-56.

23. Semiconductor Components Industries L. AR0130CS 1/3-inch CMOS Digital Image Sensor; 2018. Available from: https://www. onsemi.com/pub/Collateral/AR0130CS-D.PDF.
24. Rasti B, Scheunders P, Ghamisi P, Licciardi G, Chanussot J. Noise reduction in hyperspectral imagery: overview and application. Remote Sens 2018; 10: 482.

25. Rasti B, Ulfarsson MO, Ghamisi P. Automatic hyperspectral image restoration using sparse and low-rank modeling. IEEE Geosci Remote S 2017; 14: 2335-9. 